\title{
Degeneration of Meissner Corpuscles after Spinal Cord Transection in Rats
}

\author{
RYUJI KobaYASHI ${ }^{1}$, NAOHIKO KANEMURA ${ }^{2)}$, HirOKI KAJIHARA ${ }^{2)}$, ERIKO TSUTSUMI ${ }^{2}$,

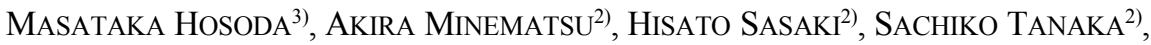 \\ KUnJI SHIRAHAMA ${ }^{2}$, JUNICHI SHIMIZU' ${ }^{2}$, KUMIKO ONO ${ }^{1)}$, OSAMU YOSHIMURA ${ }^{2)}$ \\ 1) Faculty of Rehabilitation, School of Allied Health Sciences, Kitasato University: 1-15-1 \\ Kitasato, Sagamihara, Kanagawa 228-8555, Japan.E-mail: dreamer@ahs.kitasato-uu.ac.jp \\ 2) Institute of Health Sciences, School of Medicine, Hiroshima University \\ 3) Faculty of Health Sciences, Tokyo Metropolitan University of Health Sciences
}

J. Phys. Ther. Sci.

13: 37-43, 2001

\begin{abstract}
In this study we try to clarify sequential changes in Meissner corpuscles after transection of the spinal cord. Meissner corpuscles stained with cholinesterase in the experimental group were already significantly fewer one week after spinal cord transection. The condition of rats in the first week after the operation was regarded as a spinal shock period. Denervation caused a decrease in the number of stained Meissner corpuscles in the shock period. In the experimental group at 7 weeks after the operation, atrophy of these corpuscles was statistically significant. These rats could not carry their weight on their hind limbs, and this change was regarded as the effect of disuse. The results of this study suggest the possibility of human sensory organs' atrophy after spinal cord injury.
\end{abstract}

Key words: Degeneration, Meissner corpuscle, Spinal cord transection.

(This article was submitted Jun. 29, 2000, and was accepted Sep. 20, 2000)

\section{INTRODUCTION}

A sensory nerve ending which senses exterior stimulation through the dynamic transformation of the receptor is called a mechanoreceptor. A Meissner corpuscle is one of the mechanoreceptors that exists in the dermal papillae. It works to receive dynamic touch and pressure sense $\mathrm{e}^{1,2)}$. The cytological structure of this corpuscle and hystochemical nature in humans is thought to be homologous with those of rats $^{3-5}$.

Meissner corpuscles exist at a higher density than any other receptor and have several innervations per corpuscle. Thus, they have a close relation to fine tactile gnosis and postural adjustment as well as the beginning of earlier recovery from trauma, and are therefore the most important target of sensory rehabilitation. In regard to basic research, however, a thorough report has not been completed regarding Meissner corpuscles morphological changes after spinal cord injury.

It is supposed that the spinal cord bears a part in motor programming according to a distributed motor control model ${ }^{6)}$ submitted recently. It has been shown in animal experiments that the spinal cord, when severed from the brain, acquired the ability of movement required for walking ${ }^{7}$. If the motor learning of the spinal cord and the effector organ below the injured part is advanced, it might be indispensable to know when the change in the mechanoreceptor occurs to decide when to begin rehabilitation training.

Therefore, the significance of this study was to initiate basic research on the sensory organ, below the injured part, after spinal cord injury. The primary purpose of this study was to clarify changes 
in the Meissner corpuscle by making time interval observations after spinal cord transection using nonspecific cholinesterase activity as a parameter.

\section{MATERIALS AND METHODS}

\section{Materials}

Thirty-two Wistar male rats (about $180 \mathrm{~g}$ body weight) purchased at the age of 5 weeks were used. They were given an adjustment period of one week in the experimental laboratory. Of them, 20 were allotted as the experimental group and 12 underwent a sham operation (control group). The temperature in the laboratory was kept at $22^{\circ} \mathrm{C}$ and lighting was turned on every 12 hours. Water and food were available ad libitum.

\section{Operation procedure}

Rats in the experimental group were administered Nembutal ${ }^{\circledR}$ (sodium pentobarbital $5 \mathrm{mg} / 100$ g body weight) as anesthesia by intraperitoneal injection. After skin incision of the back, the thoracic spine was exposed at the height of the inferior angle of the scapula. The spinal cord was exposed by laminectomy at level Th6-8 and was transected with a scalpel. If the cut seemed incomplete, it was severed completely with tweezers. In each case, the completeness of the transection was confirmed by depression of the muscle tone of the lower limb and by no reaction to stimuli by a pin against the planta. The spinal cords of rats in the control group were exposed but not transected.

\section{Behavior observation}

Rats were put into a standard chip cage and allowed free activity using their forelimbs for locomotion. To alleviate the possibility of reduction in the range of joint motion, the hindlimbs of each spinalized rat was passively manipulated through a normal range of motion once a day.

Movements of the rats were observed every day. Behavior assessment is a modified version of the test protocol described by Kerasidis ${ }^{8)}$. The test for each animal lasted for 10-15 $\mathrm{min}$, and the following protocol was routinely used:

a. Movement of the hind limbs.

The rat was placed in a large open field and utilization of the hindlimbs in locomotion was observed and evaluated. Each hindlimb was observed separetely and classified as follows: no movement of hindlimb, and no weight bearing; barely perceptible movement of hindlimb, and no weight bearing; frequent and/or vigorous movement of hindlimb, and no weight bearing; hindlimb can support weight, may take a few steps; walks with only mild deficit; normal walking.

b. Muscle tone of the hind limbs.

The rat was held down on a table by the observer's left hand around the rat's torso. A hindpaw was held gently between the observer's right thumb and forefinger and moved passively to the full range of the hindlimb. Each hindlimb was observed separately and classified as follows: flaccid; mild spastic tone; moderate spastic tone; hyper tone; normal.

c. Pain withdrawal reflex.

The nociceptive responses of the hindquarters were evaluated on the planta by pain stimuli. The rat was held down on a table on its side so that the hindlimb to be tested was uppermost. The foot was then briefly pinched with a clothespin. Withdrawal reflexes were classified as follows: no withdrawal; weak withdrawal; normal withdrawal; hyperactive withdrawal.

\section{Enzyme histochemical procedure}

With the exception of one rat which died during the experimental period, each of the experimental and control rats were divided into 4 groups; 1 week, 3 weeks, 5 weeks and 7 weeks after operation (Table 1). The hind toe pads were divided into A, B, C, D, $\alpha$ and $\beta$ region based on Kennedy's approach $^{9)}$ (Fig. 1). The B and C pads were resected from both hindlimbs under anesthesia. One toe pad was ulcerated and was excluded (Table 1). At sacrifice, a lethal dose of anesthesia was injected into the abdomen.

Biopsied samples were fixed in cold $10 \%$ formalin solution (including $1 \% \mathrm{CaCl})^{2}$. After washing the tissues, they were fixed on cork using tragacanth gum and quick frozen by isopentane cooled with liquid nitrogen. Frozen tissues were cut into $10-\mu \mathrm{m}$ sections with a cryostat. One part was sectioned transversely against the long axis at the center of the toe pad. Histochemistry was conducted by Karnovski \& Roots's method ${ }^{10)}$. After being air dried, specimens were soaked in an incubation medium at $37^{\circ} \mathrm{C}$ for $30 \mathrm{~min}$. Acetylthiocholine iodide (Sigma Co.) was used as a substrate, and the non-specific cholinesterase active site was stained brown. In order for the stainings to be done under the same conditions, the samples of 
Table 1. The number of samples.

\begin{tabular}{lccc}
\hline Group & Number of animals & Toe pad (no ulcer) & Toe pad (ulcerated) \\
\hline 1 week control & 3 & 12 & 0 \\
1 week experimental & 5 & 20 & 0 \\
3 weeks control & 3 & 12 & 0 \\
3 weeks experimental & 5 & 20 & 0 \\
5 weeks control & 3 & 12 & 0 \\
5 weeks experimental & 5 & 20 & 0 \\
7 weeks control & 3 & 12 & 0 \\
7 weeks experimental & 4 & 15 & 1 \\
exception for death & 1 & & \\
\hline
\end{tabular}
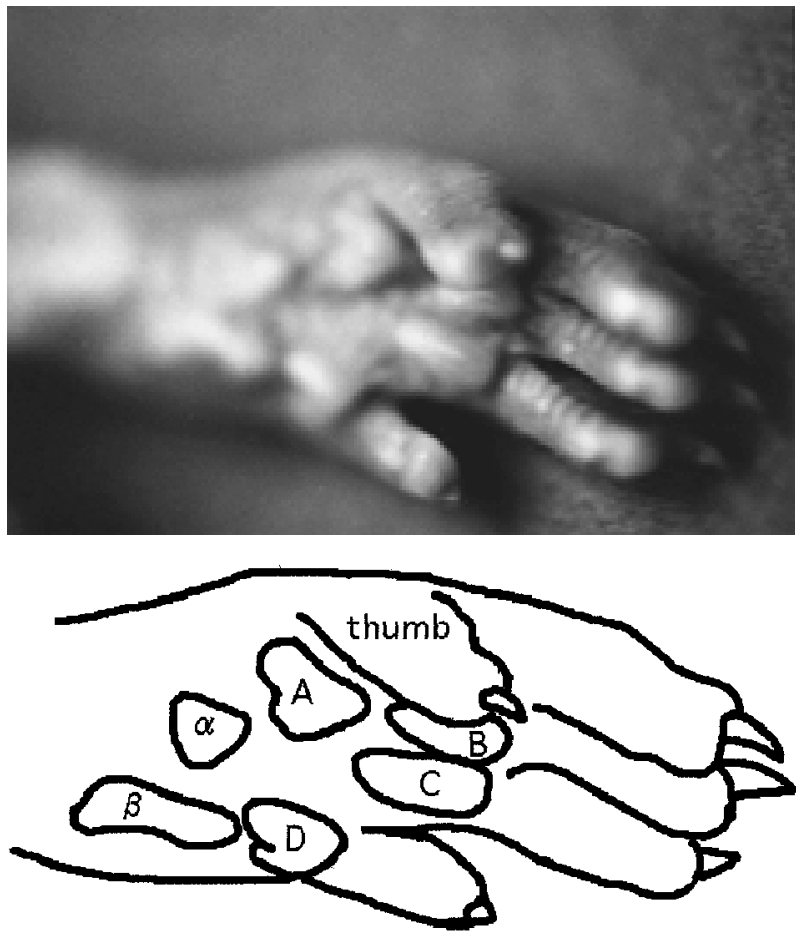

Fig. 1. The rat's hind toe pads. Kennedy ${ }^{3)}$ classified toe pads. $\mathrm{B}$ and $\mathrm{C}$ were resected in this study.

each group were taken in the same week and soaked in the same reaction solution. After the processes of rinse, alcohol dehydration, xylene penetration and mounting with Eukit (O. Kindler Co.), the samples were observed under an optical microscope.

\section{Morphometric analysis}

A computerized morphometric analyzer was used to determine population density and extent of atrophy of Meissner corpuscles. Recognizable stained Meissner corpuscles were counted and photographed with reversal film (Fuji PROVIA). After scanned by a film-scanner (Polaroid Co.), the image was put in a personal computer (Macintosh) by image processing software (Photoshop Ver.2.5). The acquired TIFF image was processed by morphometry software (NIH Image Ver.1.61) to attain the data of the stained area for every Meissner corpuscle. The stained area was extracted by adjustment of the Density Slice function in NIH Image and was measured.

A two-way analysis of variance (ANOVA) was used to test the effects of spinal transection, the spinalized duration on the number of Meissner corpuscles, and the stained area. The alpha level for the ANOVA test was 0.05. All statistical analyses were performed using JavaScript-STAR Version $0.9 \mathrm{~b} 5$ computer software package.

\section{Approval of experiment}

This study was approved by the animal experiment institution attached to the Hiroshima University School of Medicine and was carried out according to the animal experiment ethical category of Hiroshima University.

\section{RESULTS}

\section{Behavior observation for the experimental group}

a. For the first week after spinal cord transection

Rats with transected spinal cords showed flaccid paralysis and dragged their hind limbs as they walked and moved with only fore limbs. Throughout the entire experiment, their plantas tended to face upward. Therefore they did not support their weight by touching their plantas on the ground (Fig. 2). Reflex movement in the lower limbs had disappeared within 3 or 4 days after the operation although at the end of this period, 
withdrawal reflex caused by pain stimuli appeared clearly.

b. Two and three weeks after spinal cord transection

During this period, when the animals moved forward, a total flexion pattern of hind limbs on the trunk concave side and an extension pattern of hind

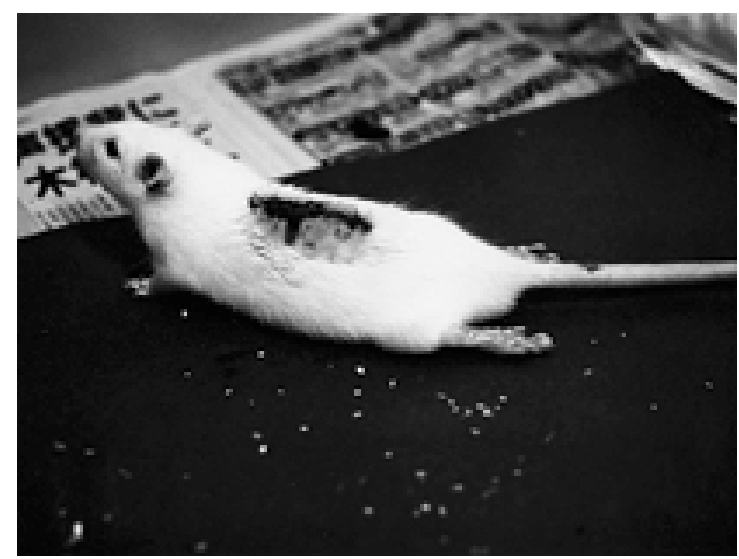

Fig. 2. Spinal cord transected rat. During the entire period of the experiment, their plantas tended to face upward. limbs on the convex side appeared. Reflex movement became remarkable and crossed extension reflex was induced by nociceptive stimulation. Muscles of the hind limbs changed from flaccid to normal, or slightly spastic tonus.

c. Four and five weeks after spinal cord transection

Movements of the hind limbs were only synergetic flexion-extension and still showed no selective movement. Withdrawal reflex also did not differ from that in the previous period. However, muscle tone showed hypertonicity compared with the control group.

d. Six and seven weeks after spinal cord transection

The planta still tended to face up without supporting the animal's own weight. Some toes had dried planta skin or ulcers had formed. Movement was the same as for the previous period.

\section{Microscopic observation}

In non-specific cholinesterase staining, Meissner corpuscles proved positive and became dark-brown (Fig. 3a). Those sites were particularly dark in color. However, in the experimental group at 7
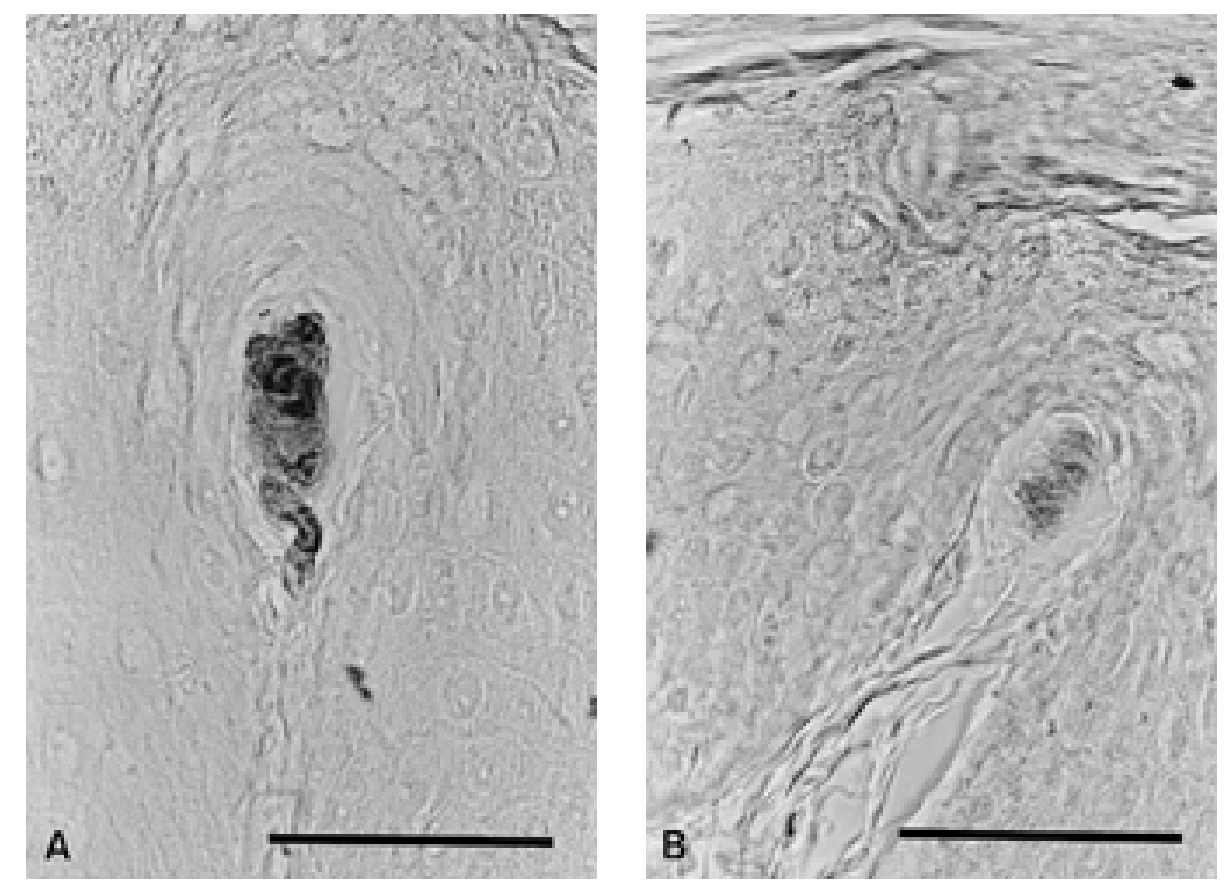

Fig. 3. 7 weeks after the operation (non-specific cholinesterase staing $\times 200, \mathrm{BAR}=50 \mu \mathrm{m}$ ). A. Meissner corpuscle of the control group proved positive and became dark-brown. B. In the operation group, atrophic corpuscles were clearly observed. 
Table 2. Means and standard deviations for the number of stained Meissner corpuscles

\begin{tabular}{lcccc}
\hline Variables & 1 week & 3 weeks & 5 weeks & 7 weeks \\
\hline Control group & $4.75 \pm 1.66$ & $3.00 \pm 1.54$ & $3.58 \pm 1.78$ & $4.17 \pm .33$ \\
Experimental group & $3.00 \pm 2.15$ & $2.05 \pm .57$ & $3.05 \pm .31$ & $2.67 \pm 1.59$ \\
\hline
\end{tabular}

Values are means $\pm \mathrm{SD}$.

Table 3. Results of two-way ANOVA for the number of stained Meissner corpuscles

\begin{tabular}{lrrrc}
\hline \multicolumn{1}{c}{ Source } & df & \multicolumn{1}{c}{ SS } & \multicolumn{1}{c}{ MS } & \multicolumn{1}{c}{ F } \\
\hline Weeks & 3 & 28.26 & 9.42 & \multicolumn{1}{c}{2.57} \\
Spinal transection & 1 & 40.74 & 40.74 & $11.10^{*}$ \\
Weeks $\times$ spinal transection & 3 & 6.65 & 2.22 & 0.60 \\
Error & 115 & 422.07 & 3.67 & \\
\hline
\end{tabular}

* Significant at $\mathrm{P}<0.05$.

Table 4. Means and standard deviations for the stained area $\left(\mu \mathrm{m}^{2}\right)$ of Meissner corpuscles.

\begin{tabular}{lcccc}
\hline Variables & 2 weeks & 3 weeks & 5 weeks & 7 weeks \\
\hline Control group & $2204 \pm 1211$ & $2420 \pm 2214$ & $2511 \pm 1154$ & $2790 \pm 1778$ \\
Experimental group & $2323 \pm 1456$ & $2262 \pm 1287$ & $218 \pm 1539$ & $1579 \pm 83$ \\
\hline
\end{tabular}

Values are means \pm SD.

weeks after operation, atrophic corpuscles were clearly observed as well as obvious degeneration and a remarkable decline of staining (Fig. 3b).

\section{Data comparison between control and experi- mental groups}

The number of samples which were dealt with statistically are shown in Table 1.

a. Comparison of the number of Meissner corpuscles between experimental and control groups

Group means and standard deviations for the number of stained Meissner corpuscle are shown in Table 2. The two-way ANOVA revealed no difference at $\mathrm{p}<0.05$ level between the weeks (Table 3 ), but ANOVA showed that the main effect was that the number of Meissner corpuscle after spinal transection decreased significantly. There was no weeks $\times$ spinal transection interaction.

b. Comparison of area of Meissner corpuscle between experimental and control group

Group means and standard deviations for the area of stained Meissner corpuscles are shown in Table 4. The two-way ANOVA revealed a weeks $\times$ spinal transection interaction (Table 5). A "simple main effects" of time factor was demonstrated in 7 weeks. Thus, the area of Meissner corpuscle of the experimental 7 weeks group was reduced significantly.

\section{DISCUSSION}

Our results showed that the number and area of Meissner corpuscles decreased. There are many factors, both neural and those related to mechanical immobilization, behind mechanoreceptor degeneration. Iida and Tachibana ${ }^{11)}$ reported an increase in regressive Meissner corpuscles as animals advanced in age. Peripheral denervated Meissner corpuscles also became smaller and hardly identifiable ${ }^{12,13)}$. Michinaka et al. ${ }^{14)}$ showed increases in atrophic mechanoreceptors after immobilization of the knee joint. Morphological and histological evidence of degeneration of mechanoreceptors in meniscus after meniscectomy were reported by Fairbank ${ }^{15)}$. However, little is known concerning changes of mechanoreceptors after spinal cord transection. 
Table 5. Results of two-way ANOVA for the stained area $\left(\mu \mathrm{m}^{2}\right)$ of Meissner corpuscles

\begin{tabular}{lrrrc}
\hline Source (simple main effect) & df & \multicolumn{1}{c}{ SS } & MS & F \\
\hline Weeks & 3 & 1637773 & 545924 & 0.25 \\
$\quad$ (weeks at control) & 3 & 8203116 & 2734372 & 1.26 \\
$\quad$ (weeks at experimental) & 3 & 16449704 & 5483235 & 2.53 \\
& & & & \\
Spinal transection & 1 & 14529291 & 14519291 & $6.72^{*}$ \\
$\quad$ (transection at 1 week) & 1 & 328254 & 328254 & 0.15 \\
(transection at 3 weeks) & 1 & 590489 & 590489 & 0.27 \\
(transection at 5 weeks) & 1 & 2522235 & 2522235 & 1.17 \\
(transection at 7 weeks) & 1 & 34103360 & 34103360 & $15.76^{*}$ \\
& & & & \\
Weeks $\times$ spinal transection & 3 & 23015047 & 7671682 & $3.55^{*}$ \\
Error & 378 & 817824223 & 2163556 & \\
\hline
\end{tabular}

* Significant at $\mathrm{P}<0.05$.

Our histological study showed a dramatic decrease in the number of mechanoreceptors within 1 week. The behavior observation showed that the condition of rats one week after the operation in this experiment was regarded as a period of spinal shock. If a muscle is flaccidly paralysed, which is the case in acute spinal cord injuries due to spinal shock, both efferent (motor) and afferent (sensory) innervation degenerate ${ }^{16)}$. Ide ${ }^{12)}$ previously deduced from a denervation experiment that maintenance of normal morphology of the terminal Schwann cell of the mouse Meissner corpuscle and their synthesis of ChE protein are dependent on the axon terminal. Thus, the decrease of Meissner corpuscles might be due to nerve degeneration.

With spinal shock behind them, rats regained reflex activity and synergic movement gradually. When nerves came into play, the remaining Meissner corpuscles seemed to work again. Thus we consider that the area atrophy of Meissner corpuscles depends on the disuse. In fact, rats which had their spinal cords cut did not support their weight with their hind limbs. After the operation, they dragged themselves and their plantas tended to face upward. According to Michinaka et al. ${ }^{14)}$, lack of physical stimulation due to disuse led the receptor in the ligament to atrophy.

Ide $^{12)}$ showed that surgically-induced sensory denervation could atrophy the mechanoreceptor totally. In our study, atrophy was not shown in all corpuscles. We think that this degeneration differed from the uniform atrophy of corpuscle because of the cutting of the peripheral nervous system.

Since spinal cord injury occurs in a proportion of
40 people to a population of one million ${ }^{17)}$, it is one of the important object injuries for rehabilitation. Success of some reconstructional approach to the paralysed part after spinal cord injury, which has been ignored so far, may depend greatly on the normality of sensory input. The result of this study suggests the possibility of the human sensory organs' atrophy soon after the spinal cord injury. However, there are still many points that remain unclear. Among them is: would morphology maintenance be enabled by sensory rehabilitation? This is a topic for future studies.

\section{ACKNOWLEDGMENT}

We would like to thank Yukiko Hidaka, Syoji Miyata and Mai Kobayashi for their help in these investigations.

\section{REFERENCES}

1) Ide C: Mechanoreceptors: skin. 1996, Seitai no kagaku, 47: 490-493 (in Japanese).

2) Munger BL, Ide $C$ : The structure and function of cutaneous sensory receptors. 1988, Arch Histol Cytol, 51: $1-34$.

3) Hurley HJ, Mescon H: Localization of non-specific cholinesterase in Meissner's corpuscles in human skin. 1956, Br J Dermatol, 68: 290-293.

4) Cauna N: The distribution of cholinesterase in the cutaneous receptor organs, especially touch corpuscles of the human fingers. 1960, J Biophys Biochem Cytol, 8: 467-482.

5) Munger BL: Neural-epithelial interactions in sensory receptors. 1077, J Invest Dermatol, 69: 27-40.

6) Horak FB: Assumptions underlying motor control for 
neurologic rehabilitation. In: Lister MJ (ed) Contemporary management of motor control problems. Proceedings of the II STEP conference. 1991, Virginia, Foundation for Physical Therapy, pp 11-21.

7) Hodgson JA, Roy RR, Leon R, et al.: Can the mammalian lumbar spinal cord learn a motor task? 1994, Med Sci Sports Exerc, 26: 1491-1497.

8) Kerasidis H, Wrathall JR, Gale K: Behavioral assessment of functional deficit in rats with contusive spinal cord injury. 1987, J Neuroscience Methods, 20: 167-189.

9) Kennedy WR, Sakuta M, Quick DC: Rodent eccrine sweat glands : a case of multiple efferent innervation. 1984, Neuroscience, 11: 741-749.

10) Karnovsky MJ, Roots LA: "Direct-coloring" thiocholine method for cholinesterase. 1964, J Histochem Cytochem, 12: 219-221.

11) Iida $S$, Tachibana $T$ : Age related changes in Meissner corpuscles in the mouse palate: a histochemical and ultrastructural study. 1996, Arch Histol Cytol, 59:
281-290

12) Ide C: Degeneration of mouse digital corpuscles. 1982, Am J Anat, 163: 59-72.

13) Navarro $X$, Verdu E, Wendelschafer-crabb G, Kennedy WR: Immunohistochemical study of skin reinnervation by regenerative axons. 1997, J Comparative Neurol, 380: 164-174.

14) Michinaka $Y$, Yamamoto $H$, Kawakami $T$, et al.: Effect of immobilization of knee joint on mechanoreceptors in anterior cruciate ligament of the rabbit. 1997, J Orthop Sci, 2: 259-265.

15) Fairbank TJ: Knee joint changes after meniscectomy. 1948, J Bone Joint Surg, 30: 664-670.

16) Jozsa L, Kannus P, Jarvinen TAH, et al.: Number and Morphology of mechanoreceptors in the myotendinous junction of paralysed human muscle. 1996, J Pathology, 178: 195-200.

17) Singu H: Spinal cord injuries in Japan: a nationwide epidemiological survey in 1990. 1994, Paraplegia, 32: 3-8. 